\section{THE SERENDIPTICHORD: REFLECTIONS ON THE COLlABORATIVE DESIGN PROCESS BETWEEN ARTIST AND RESEARCHER}

Tim Murray-Browne, Di Mainstone, Nick Bryan-Kinns and Mark D. Plumbley, Queen Mary University of London, Centre for Digital Music, London, E1 4NS, U.K. E-mail: $<$ tim.murraybrowne@elec.qmul.ac.uk>, $<$ dimainstone@hotmail.com>.

See $<$ www.mitpressjournals.org/toc/leon/46/1> for supplemental files associated with this issue.

Submitted: 1 April 2012

\section{Abstract}

The Serendiptichord is a wearable instrument, resulting from a collaboration crossing fashion, technology, music and dance. This paper reflects on the collaborative process and how defining both creative and research roles for each party led to a successful creative partnership built on mutual respect and open communication. After a brief snapshot of the instrument in performance, the instrument is considered within the context of dance-driven interactive music systems followed by a discussion on the nature of the collaboration and its impact upon the design process and final piece.

\section{As the lights dimmed before a packed} audience of conference goers in the auditorium of Berkeley Art Museum, a large black box took centre stage, haloed with light from above. Dancer Heidi Buehler subsequently appeared onstage, and with an air of mischievous curiosity proceeded to open the solitary container. One by one she extracted the red modular elements that make up the Serendiptichord. As she liberated the curvaceous leather headpiece from the box the audience became aware of the link between her manipulation of the object and shards of sound filling the auditorium. Intrigued by her newfound influence, she then investigated each component on and around the body, releasing ribbons of sound with every move. When she allowed the instrument to slip over her shoulders, she experienced its power and its possessive nature through to a vociferous climax. Realizing its seemingly irresistible domination she tore it off her body, hurling it into the box. The Serendiptichord, now tranquil, seemed enticing once more but Heidi resisted, sealing the box from which it came.

The Serendiptichord (Fig. 1) is a wearable musical instrument designed to entice the user to explore a soundscape through touch and movement [1]. It is the result of a cross-disciplinary collaboration between Mainstone, an artist who combines technology, fashion and per-

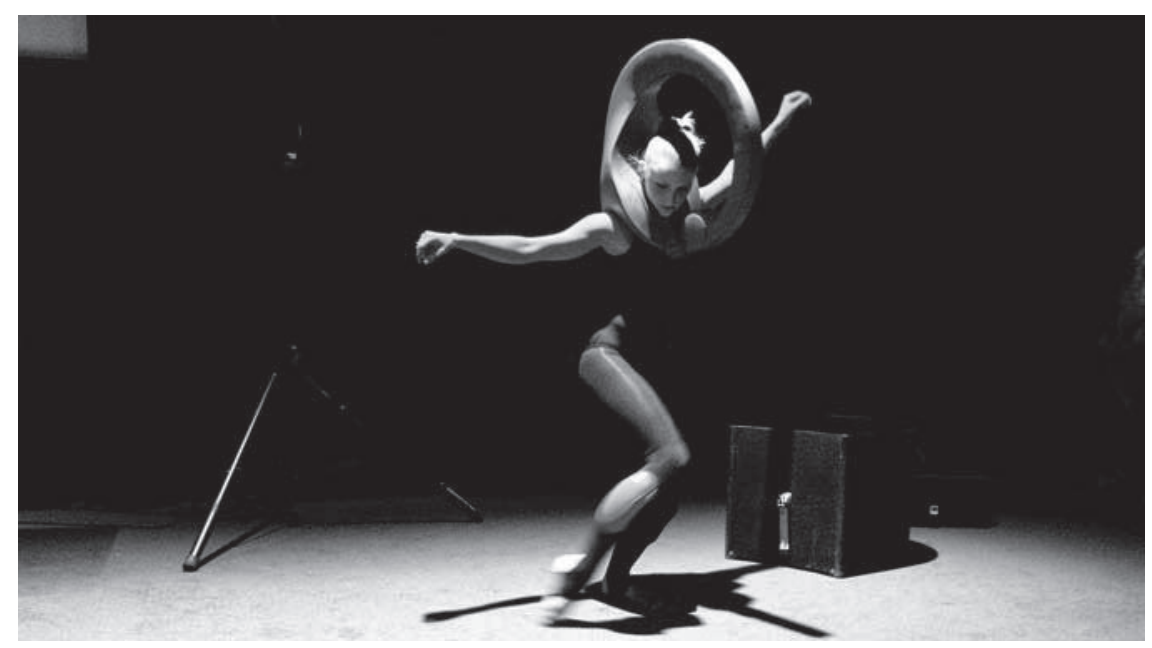

Fig. 1. Heidi Buehler with the Serendiptichord at the ACM Creativity \& Cognition Conference 2009. (C Di Mainstone and Tim Murray-Browne. Photo: Deirdre McCarthy.)

formance, and Murray-Browne, a $\mathrm{PhD}$ candidate investigating the use of narrative within interactive music systems.

Performances incorporating generative sonic interpretation of dance have been undertaken with a number of different techniques and motivations. Camurri et al. [2] developed a platform which uses a camera to interpret a dancer's gestures, shape and emotional intention. Different mappings were developed alongside dancers and composers in a summer workshop for a concert combining musicians, dancers and generative sound. As an alternative to computer vision, wireless motion sensors have been used in systems such as [3] and Sensemble [4], a multi-user system drawing on correlations of movement between dancers.

Work produced for these systems is often driven by a desire to demonstrate the possibilities of a sensing technology (e.g. [4]). In contrast, this project drew on current research into different conceptual approaches to interactive music systems with an aim to explore its possibilities within current artistic practice. Consequently, development was art-led rather than technology-led [5: 62] in that technology was developed to satisfy artistic goals rather than art developed to explore new sensing methods.

\section{The Collaborative Process}

The initial purpose of this collaboration was both to further artistic practice and to transfer knowledge between different fields. However, by not adhering to the traditional roles apportioned to artist and researcher, some of the common pitfalls of an artist/assistant relationship [5: 61] were avoided. From the outset, Mainstone's extensive background of sculptural, tactile and interactive work and Murray-Browne's in sound installation led to distinct creative roles overseeing the sculptural and sonic aspects of the work. Similarly, having spent a number of years in a research environment combining art and technology,

Mainstone's role is of researcher as well as artist. Consequently, each party took great interest in the other's work, desiring to learn how it may play a part in future work.

Early communication between the collaborators chiefly focused on how each other's ideas related, technical feasibility and indications of preference among developing themes, allowing an understanding of the issues and possibilities to develop openly. Where these areas met - conceptual development and interaction design - a greater amount of collaborative brainstorming took place, with the artist suggesting how physical components might sound and the researcher how sounds might be invoked.

As in any artistic project, ideas were often pursued or dropped based on intuition informed by past experience and personal objectives and tastes. Whilst not blocking this process, collaborating shaped it by requiring decisions to be explicitly justified, enforcing a form of intellectual rigour.

\section{Developing the Concept}

Murray-Browne came to the project aiming to apply his research on the place of narrative (i.e. macroscopic structure) within interactive music systems: how can a composer both develop musical ideas and provide interactional freedom? The enticing and provoking nature of Mainstone's work offered potential to address this dichotomy by coaxing a listener through a canonical narrative. 
Mainstone arrived with a series of concepts referencing sound, exploratory movement and connection within public space (Fig. 2), a direct response to her current work in New York. As the collaboration developed these themes filtered, focusing on the sense of movement, narrative and sound. Both parties shared a fascination with narrative. Mainstone uses story to develop a physical architecture around the body as well as to create potential for interactive scenarios, which complemented MurrayBrowne's research on the place of narrative when a soundscape is explored in a non-linear fashion.

The first month of the collaboration took place with Mainstone in New York and Murray-Browne in London through Skype and 'PowerPoint ping-pong'-a virtual sketchpad emailed back and forth. Key words like narrative, exploration and space would resonate with both parties, suggesting where the two disciplines might converge. Developing an underlying concept in terms of both artistic and research ambitions allowed both of these aspects to inform each other. For example, the need for an instrument to be predictable [6] coupled with the desire for serendipitous exploration suggested a relationship between user and instrument akin to collaborative improvisation. This led to the instrument being thought of as a character with personality traits like unrestrainable, playful, illusive, which informed aspects of the design such as the ambiguous shape or the 'animalistic' audio effect applied to the swing of the trunk. It also led to different moods being defined through sound and movement and used to construct a story between 'pioneer' (our user) and object.

Concurrently, technical restrictions were considered and informed by the developing concept. A vision of serendipitous exploration made technology overly sensitive to environmental factors (e.g. computer vision) less appealing. Furthermore, the characterization of the instrument suggested potential for dialogical interaction, which seemed more suited to a physical object that connected with the body as the origin of the sound.

\section{Design and Construction}

Both object and software were constructed simultaneously in close collaboration and small iterations. Keeping with the theme of serendipity, there was the aspiration that the instrument might be used in ways beyond its creators' intentions, which required the interaction design to be as unprescriptive as possi-

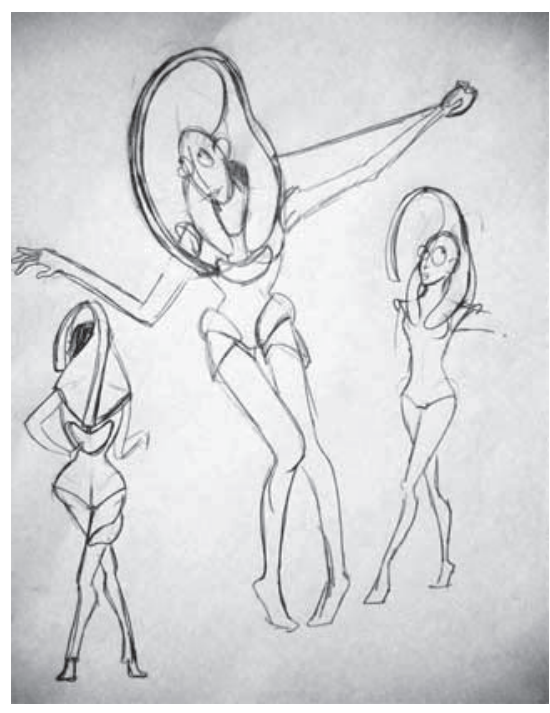

Fig. 2. Sketches drawn during the conceptual development. (C Di Mainstone)

ble. This required it to be intuitive, a demand tackled through embodied metaphors - metaphors of interaction as an extension of normal life [7] — created through body-centric development.

This approach to creating interactive art developed through Mainstone's previous work Sharewear [8], a piece which evolved through creating modular components and observing how others used them. By keeping designs open-ended, users are encouraged to follow their intuition, which the artist may observe and learn from as the work evolves.

The Serendiptichord was created around the body, guided by how its wearer felt it should feel and sound when moved and what further movements it should respond to. The wearer was influenced by the current shape and sounds of the instrument. This feedback allowed the aesthetic to evolve through short iterations of develop-and-test. Thus, the shape was defined through movement.

The mapping from movement to sound developed similarly with a more complex metaphor of exploring a 'space of concepts' being successively simplified to one of moving into sounds [9]. This resulted in less control over the organiza tion of sounds. However, the instrument's shape and physical response suggested further forms of interaction: detachable pods that could attach to parts of the body and an overlaid effect controlled by the natural swing of the 'trunk' (see Fig. 1).

At the first opportunity an open workshop was held where visitors could play and experiment with the Serendiptichord as part of London's Inside Out festival. Time constraints meant the instrument was shown with the upholstery halfcomplete and subject to 'live debug- ging.' But a benefit of this informal public outing was an atmosphere of 'work in progress,' encouraging constructive and enthusiastic feedback from those present. Observation and discussion revealed that the new mapping strategy embodied a metaphor of hitting percussive objects. This fed back into the sound design: sounds 'hit harder' should be louder.

Most enlightening, however, was observing the instrument in the hands of a contemporary dancer, whose instant connection showed the piece was unquestionably for dancers. This greatly influenced subsequent development and the nature of the final performance.

\section{Conclusion}

The creation of the Serendiptichord was enhanced by both collaborators having distinct creative and technical roles.

Each took leadership over their specialist domain, with conceptual coherency maintained through short development cycles.

This design process was enhanced by a short deadline which brought both a clarity of vision - sometimes clouded in longer research projects - and the alignment of each element that made up the creative process, meaning that both design and technical issues were addressed at every stage of development.

\section{References and Notes}

1. Commissioned by the Centre for Digital Music grant EPSRC EP/E045235/1 for the $A C M C \& C$ Conference 2009, produced by BigDog Interactive, supported by the Interactional Sound and Music Group and an EPSRC Doctoral Training Account. Thanks to Rachel Lamb, Judy Zhang, Stacey Grant and Vesselin Iordanov.

2. A. Camurri, B. Mazzarino and G. Volpe, "Expressive gestural control of sound and visual output in multimodal interactive systems," in Proc. ICMC'04 (2004).

3. C. Park, P.H. Chou and Y. Sun, "A wearable wireless sensor platform for interactive art performance," in Proc. IEEE PerCom'06 (2006) pp. 1317.

4. R. Aylward and J. Paradiso, "Sensemble: a wireless, compact, multi-user sensor system for interactive dance," in Proc. NIME'06 (2006) pp. 134-139.

5. Linda Candy and Ernest Edmonds, Explorations in Art and Technology (London, U.K.: SpringerVerlag, 2002)

6. S. Jordà, "Instruments and players: Some thoughts on digital lutherie," J. New Music Research 33, No. 3 (2004) pp. 321-341.

7. A. Antle, G. Corness and M. Droumeva, "What the body knows: Exploring the benefits of embodied metaphors in hybrid physical digital environments," Interacting with Computers 21, No. 1-2 (2009) pp. 66-75.

8. D. Mainstone (2008), <www.v2.nl/lab/ projects/sharewear>, accessed 15 March 2010.

9. T. Murray-Browne, D. Mainstone, N. BryanKinns and M. D. Plumbley, "The Serendiptichord: A wearable instrument for contemporary dance performance," in Proceedings of the 128th Convention of the Audio Engineering Society, London, 2010. 